\title{
Monte Carlo calculation of electron relaxation times in perfect and disordered quantum wire laser structures
}

\author{
Igor Vurgaftman and Jasprit Singh \\ Department of Electrical Engineering and Computer Science, The University of Michigan, Ann Arbor, \\ Michigan 48109-2122
}

(Received 9 November 1992; accepted for publication 10 February 1993)

\begin{abstract}
We calculate the electron relaxation times in GaAs quantum wires of $100 \times 100 \AA$ and $200 \times 200$ $\AA$ cross sections by a Monte Carlo simulation with the inclusion of electron-phonon and electron-electron interactions for the wire band structure computed by solving the one-band Schrödinger equation with and without structural disorder at the wire interface. The electron relaxation time is found to be greater than a hundred picoseconds for the $100 \times 100 \AA$ wire, while it is approximately 10 ps for the wire with the $200 \times 200 \AA$ cross section with the results converging to the case of bulk material for increased wire cross section. The influence of disorder on carrier relaxation in quantum wires is found to be negligible. The unusually slow carrier equilibration process is attributed to the combined effect of the sharply peaked one-dimensional density of states and the absence of randomizing intrasubband carrier-carrier scattering processes in quasi-one-dimensional systems. The dependence of electron relaxation times on the size of the quantum wire appears to be extremely sensitive.
\end{abstract}

Quasi-one-dimensional systems are attracting considerable attention owing to their potential applications in low threshold lasers and high-speed electronic devices. ${ }^{1-7}$ The density of states in quantum wires develops a strong peak near the band edge with the promise of yielding high gains at very low injected carrier concentrations. It has been demonstrated theoretically as well as experimentally that lasers with quantum wire active regions exhibit lower threshold currents than their quantum well counterparts. ${ }^{8,9}$ The effect of fabrication-induced disorder on the density of states of a quasi-one-dimensional system has been investigated and found not to affect the band-edge singularity. ${ }^{10}$ The new band-edge states are, however, strongly localized, and it is important to identify if the disorder affects the carrier relaxation process.

The upper limit on the modulation frequency of the quantum wire laser is set by how fast the electrons injected into the quantum wire structure relax to an equilibrium distribution. The transport properties ${ }^{11-14}$ and hot-electron relaxation in quasi-one-dimensional systems for bulk ${ }^{15}$ and confined phonon modes ${ }^{16}$ have been investigated recently. The calculations were based, however, on the assumption of extreme quantum limit (i.e., electrons are confined to the lowest subband). To the best of our knowledge, no studies of carrier equilibration process in a quantum wire including band structure and scattering effects in a complete and consistent manner have been reported to date.

In this letter we study the electron relaxation processes in quantum wires of $100 \times 100 \AA$ and $200 \times 200 \AA$ cross sections. The well region material is GaAs, while the barrier region material is taken to be the wider-band-gap $\mathrm{Al}_{0.3} \mathrm{Ga}_{0.7}$ As. We calculate the electronic wave functions and the energies of the eigenstates in the wire by solving the Schrödinger equation for a single band of $s$-type states by the finite difference method. Interface disorder is introduced in the $100 \times 100 \AA$ wire to study how it affects the carrier relaxation process. A finite length along the wire axis is assumed in order to include the effects of structural disorder by randomly placing islands of either the barrier region or the well region material along one sidewall. ${ }^{10} \mathrm{We}$ have assumed a 50:50 chance of the islands on the disordered edge being $\mathrm{GaAs}$ or $\mathrm{Al}_{0.3} \mathrm{Ga}_{0.7}$ As. Above the edge of the potential well formed at the heterojunction, electrons will have a three-dimensional density of states (Fig. 1). We study electron relaxation in these structures by performing an ensemble Monte Carlo simulation. To simulate the processes occurring in a quantum wire laser, electrons are injected according to a thermal distribution at the barrier conduction band edge. The carriers then relax into the potential well of the quantum wire.

One-dimensional systems are peculiar in the sense that electron-electron collision events cannot redistribute energy and momentum between carriers in the same subband.

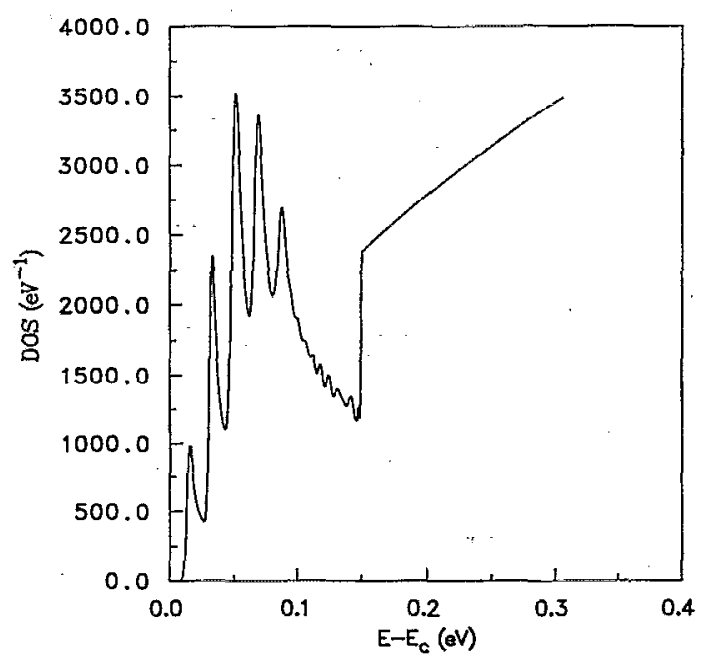

FIG. 1. The density of states calculated from the wire band structure for $200 \times 200 \AA$ quantum wire. Gaussian broadening with a linewidth of 2 $\mathrm{meV}$ is used to obtain a realistic line shape. The disorder has little effect on the density of states in the wire, and is not discernible on the scale of this figure. 
The only binary process in which energy and momentum are conserved simultaneously is an exchange of energy and momentum between the interacting electrons that has no effect on the shape of the distribution function. For wire cross sections below $200 \times 200 \AA$ the subbands are widely spaced, and the predominant energy loss mechanism is phonon scattering. The scattering rates for phonon scattering between two states $n$ and $n^{\prime}$ are given by the Fermi Golden Rule:

$$
W_{n n^{\prime}}=\frac{2 \pi}{\hbar} \sum_{\mathbf{q}}\left|M_{n n^{\prime}}(\mathbf{q})\right|^{2} \delta\left(E_{n^{\prime}}-E_{n} \pm \hbar \omega\right) .
$$

The overlap matrix element is calculated from:

$$
M_{n n^{\prime}}(\mathrm{q})=\left(n_{\mathrm{q}}+\frac{1}{2} \pm \frac{1}{2}\right)^{1 / 2} C_{\mathrm{q}} \int d^{3} r \varphi_{n^{\prime}}^{*}(\mathbf{r}) \varphi_{n}(\mathbf{r}) e^{ \pm i \mathrm{q} \cdot \mathbf{r}}
$$

where $n_{\mathrm{q}}$ is the phonon occupation number, $C_{\mathrm{q}}$ is the coupling coefficient, $\varphi_{i}$ are the envelope functions of the eigenstates, the overlap between the central cell parts of the wave functions for s-type states is unity, and the integral is over the volume of the quantum wire including the boundary region of the barrier material.

The form of the coupling coefficient for phonon scattering has been derived previously from the Fröhlich interaction Hamiltonian. ${ }^{17}$ While the importance of treating confined phonon modes in highly quantized systems has been demonstrated, ${ }^{18}$ bulk modes are a good approximation for well widths greater than $50 \AA$. A Gaussian profile of the optical phonon density of states with a linewidth of $1 \mathrm{meV}$ accounts for the dispersion of the longitudinal mode. We include the effects of screening of the phononelectron interaction at high injected carrier densities by assuming the 3D Thomas-Fermi screening potential. Acoustic phonon scattering rates are calculated using the deformation potential formalism developed for bulk modes $^{19}$ with the assumption of elasticity lifted. The scattering rates for acoustic phonons are found to be of the order of $10^{11} \mathrm{~s}^{-1}$, while the optical phonon scattering rates tend to be higher by at least an order of magnitude. Intersubband carrier-carrier processes included in this calculation for completeness are possible yet comparatively inefficient. The calculation is performed for room temperature. At lower temperatures, the occupation of phonon states will decrease with phonon emission becoming a larger fraction of scattering events. The relaxation processes will be speeded up because the energy loss mechanism becomes more efficient.

In a disordered system, low-lying electronic states are localized. In order to compute the overlap integral of the electronic wave functions with sinusoidal bulk phonon modes, we express the localized wave function as a finite series of Fourier components. Only the first four or five expansion terms at multiples of the reciprocal of the longest wavelength contribute appreciably to the overlap integral. The formalism of Ref. 17 is then applicable to both perfect and disordered wire structures. The carriers are injected in a thermal distribution at the barrier edge, and their development is followed for $50 \mathrm{ps}$ at low and high

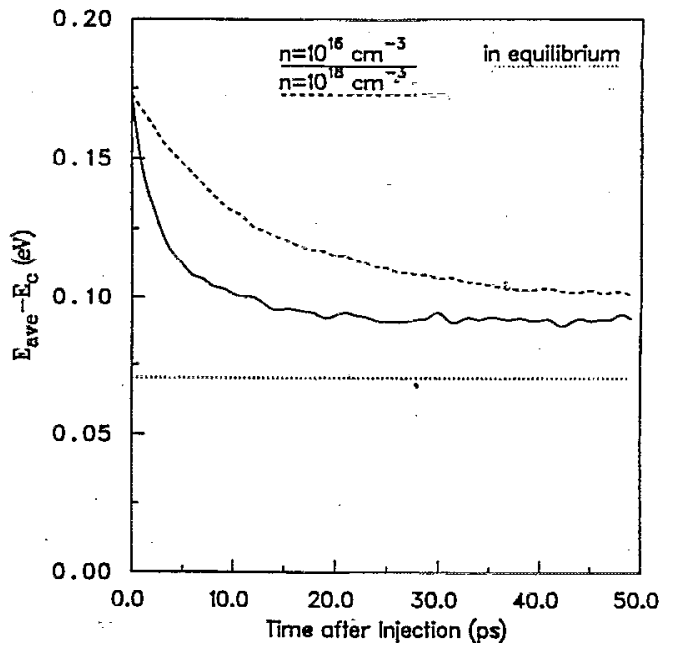

FIG. 2. The energy averaged over a large number of electrons as a function of injection time for a $100 \times 100 \AA$ quantum wire with no interface disorder for both low and high injected carrier concentrations. The horizontal line corresponds to the expectation value of the energy for the equilibrium Boltzmann distribution.

electron concentrations. At high carrier densities, the screening term in the scattering rates reduces the strength of the optical phonon-electron and electron-electron interactions considerably, while at sufficiently low concentrations, such as $10^{16} \mathrm{~cm}^{-3}$, the reduction is negligible.

Figure 2 shows the average energy of the ensemble of electrons as a function of time for the quantum wires of a $100 \times 100 \AA$ cross section for both low and high injection conditions. While initially an exponential curve may be fitted to describe carrier cooling, after approximately $10 \mathrm{ps,}$ the decrease in average energy becomes much slower. This behavior can be explained by the fact that the carriers within $36 \mathrm{meV}$ of the effective band edge, where the density of states vanishes, can give up energy only by emitting acoustic phonons, which is an inherently slow process. The effect of nonelasticity of the acoustic phonon scattering must be included to obtain correct results in this limit. We estimate the electron relaxation time to be greater than a hundred picoseconds. Note that while in the extreme quantum limit in which only one subband is occupied, the average energy of the Boltzmann distribution is $k T / 2$ above the effective band edge, the numerous peaks of the density of states function increase the expectation value of the carrier energy to $20 \mathrm{meV}$ above the effective band edge for the $100 \times 100 \AA$ quantum wire. The relaxation time is defined as the time necessary for the carrier energy to reach the expectation value of energy for the Boltzmann distribution. The results for the average energy and the electron distribution function indicate that after $10 \mathrm{ps}$ most electrons are involved in transitions between the first two subbands. The results are essentially unchanged for the disordered wire case, which can be attributed to the fact that only a few Fourier components are required to describe the localized states in a disordered quantum wire, and as a result, the overlap integral for the collision processes is not affected substantially. Moreover, the mobility edge in this quantum 


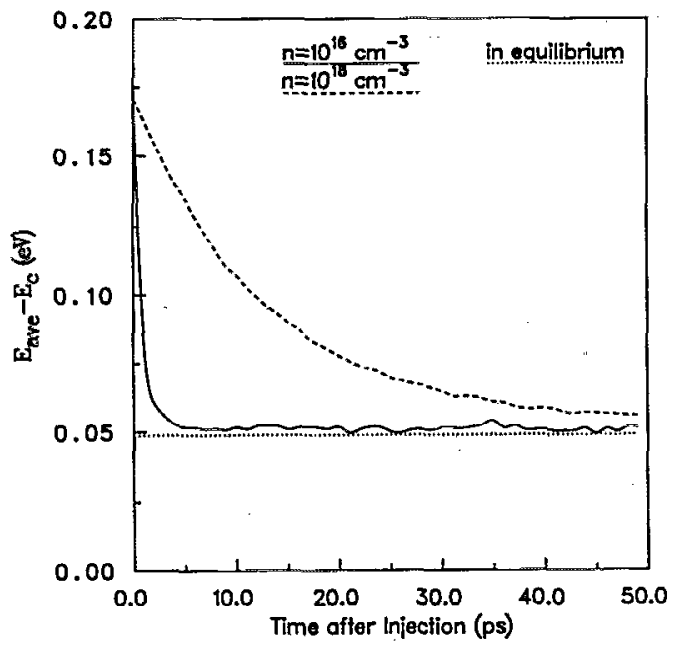

FIG. 3. The energy averaged over a large number of electrons as a function of time after injection for a $200 \times 200 \AA$ quantum wire with no interface disorder for both low and high carrier concentrations. The horizontal line corresponds to the expectation value of the energy for the equilibrium Boltzmann distribution.

wire is only $25 \mathrm{meV}$ above the effective band edge, and the majority of the electrons can scatter into these states only by acoustic phonon scattering. In the absence of screening, the optical phonon scattering rates are much higher than those at high carrier densities. Initially a greater number of carriers relax into the low-lying states of the first subband. However, the relaxation time remains of the order of a hundred picoseconds because the effect of the density of states is unaltered.

Calculations were also performed for a $200 \AA \times 200 \AA$ quantum wire. The density of states in this case exhibits many more peaks due to the increased number of subband levels (Fig. 1). The average energy for the Boltzmann distribution is increased to $35 \mathrm{meV}$ above the effective band edge, a value approximately equal to the optical phonon energy. The abnormally slow carrier cooling effect described above is not in evidence as can be seen from Fig. 3. Our results indicate that the relaxation times for a low carrier concentration are less than $10 \mathrm{ps,} \mathrm{while} \mathrm{screening} \mathrm{at}$ carrier concentrations common for semiconductor lasers increases the electron relaxation time to several tens of picoseconds. This is due to the fact that the expectation value of the equilibrium Boltzmann distribution and the phonon energy are comparable. The trend in the results is expected, since in the limit of the bulk material, the relaxation time is known to be several picoseconds.

In conclusion, we have calculated the electron relax- ation times for two sizes of quantum wire structures. For a $100 \times 100 \AA$ wire, the electron relaxation time is found to be of the order of a hundred picoseconds, a fact attributed to the sharply peaked density of states function and the absence of randomizing intrasubband carrier-carrier scattering. This conclusion is to be contrasted with results obtained by Campos et al. ${ }^{15,16}$ based on the assumption of the extreme quantum limit and zero lattice temperature and estimating the relaxation time to be of the order of several picoseconds. For a $200 \times 200 \AA$ quantum wire with six subband levels below the well potential, the electron relaxation time is calculated to be approximately $10 \mathrm{ps}$. The result is expceted to converge to that for bulk material (order of several picoseconds) for even larger wire cross sections. This shows the sensitive dependence of the electron relaxation times on the wire size. Experimental evidence available at this time does indicate substantial degradation of the small-signal response for modulation frequencies in excess of a few $\mathrm{GHz}^{8,20}$ For a V-groove quantum wire laser of Ref. 8, for example, the 3-dB modulation frequency is found to be below $10 \mathrm{GHz}$, lower than the results reported for quantum well and double heterostructure lasers. A detailed study of electron relaxation for a wide range of wire sizes is forthcoming.

This work was supported by the United States Army's URI Program (Grant No. DAAL03-87-K-0007). Discussions with Professor Y. Arakawa are greatly appreciated.

${ }^{1}$ Y. Arakawa and H. Sakaki, Appl. Phys. Lett. 40, 939 (1982).

${ }^{2}$ Y. Arakawa, K. Vahala, and A. Yariv, Appl. Phys. Lett. 45, 950 (1984).

${ }^{3}$ H. Sakaki, Jpn. J. Appl. Phys. 19, L735 (1980).

${ }^{4}$ I. Suemune and L. A. Coldren, IEEE J. Quantum Electron. QE-24, 1778 (1988).

${ }^{5}$ T. Yamauchi, Y. Arakawa, and J. N. Schulman, Appl. Phys. Lett. 57, 1224 (1990).

${ }^{6}$ Y. Arakawa, T. Yamauchi, and J. N. Schulman, Phys. Rev. B 43, 4732 (1991).

${ }^{7}$ T. Takahashi and Y. Arakawa, IEEE J. Quantum Electron. QE-27, 1824 (1991).

${ }^{8}$ S. Tiwari, G. D. Pettit, K. R. Milkove, R. J. Davis, J. M. Woodall, and F. Legoues, IEDM Proc. 852 (1992).

${ }^{9}$ I. Vurgaftman, J. M. Hinckley, and J. Singh (unpublished).

${ }^{10} \mathrm{~J}$. Singh, Appl. Phys. Lett. 59, 3142 (1991).

${ }^{11}$ S. Briggs and J. P. Leburton, Phys. Rev. B 38, 8163 (1988).

${ }^{12}$ S. Briggs and J. P. Leburton, Superlattice Microstruct. 5, 145 (1989).

${ }^{13}$ J. P. Leburton, S. Briggs, and D. Jovanovic, Superlattice Microstruct. 8, 209 (1990).

${ }^{14}$ J. P. Leburton, Phys. Rev. B 45, 11022 (1992).

${ }^{15}$ V. B. Campos and S. Das Sarma, Phys. Rev. B 45, 3898 (1992).

${ }^{16}$ V. B. Campos, S. Das Sarma, and M. A. Stroscio, Phys. Rev. R. 46, 3849 (1992).

${ }^{17}$ J. P. Leburton, J. Appl. Phys. 56, 2850 (1984).

${ }^{18}$ M. Stroscio, Phys. Rev. B 40, 6428 (1989).

${ }^{19}$ B. K. Ridley, Quantum Processes in Semiconductors (Clarendon, Oxford, 1982).

${ }^{20} \mathrm{M}$. Walther, E. Kapon, J. Christen, D. M. Hwang, and R. Bhat, Appl. Phys. Lett. 60, 521 (1991). 